\title{
Abnormal Cerebral Perfusion Profile in Older Adults with HIV-Associated Neurocognitive Disorder: Discriminative Power of Arterial Spin-Labeling
}

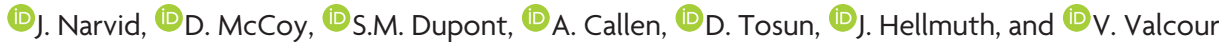

\begin{abstract}
BACKGROUND AND PURPOSE: The aging HIV-infected $\left(\mathrm{HIV}^{+}\right)$population has increased vascular comorbidities, including stroke, and increased cognitive deficits compared with the general population. Arterial spin-labeling is a technique to measure cerebral blood flow and is more sensitive than regional volume loss in assessing neurodegenerative diseases and cognitive aging. Previous studies have found global cerebral perfusion abnormalities in the $\mathrm{HIV}^{+}$participants. In this study, we evaluated the specific regional pattern of CBF abnormalities in older $\mathrm{HIV}^{+}$participants using quantitative whole-brain arterial spin-labeling.
\end{abstract}

MATERIALS AND METHODS: CBF data from the UCSF HIV Over 60 Cohort and the Alzheimer Disease Neuroimaging Initiative were retrospectively evaluated to identify $19 \mathrm{HIV}^{+}$older adults, all with plasma viral suppression (including 5 with $\mathrm{HIV}$-associated neurocognitive disorder); 13 healthy, age-matched controls; and 19 participants with early mild cognitive impairment. CBF values were averaged by ROI and compared among the 3 groups using generalized linear models.

RESULTS: When we accounted for age, education, sex, and vascular risk factors, the $\mathrm{HIV}^{+}$participants demonstrated alterations in regional cerebral perfusion, including hypoperfusion of bilateral temporal, parietal, and occipital brain regions compared with both clinically healthy participants and those with mild cognitive impairment. Arterial spin-labeling showed reasonable test characteristics in distinguishing those with HIV-associated neurocognitive disorder from healthy controls and participants with mild cognitive impairment.

CONCLUSIONS: This study found specific CBF patterns associated with HIV status despite viral suppression—data that should animate further investigations into the pathobiologic basis of vascular and cognitive abnormalities in HIV-associated neurocognitive disorders.

ABBREVIATIONS: ADNI = Alzheimer Disease Neuroimaging Initiative cohort; ASL = arterial spin-labeling; GLMnet = generalized linear model via penalized maximum likelihood; $\mathrm{HAND}=\mathrm{HIV}$-associated neurocognitive disorders; $\mathrm{HC}=$ healthy controls; $\mathrm{HIV}^{+}=\mathrm{HIV}$-infected; $\mathrm{MCl}=$ mild cognitive impairment; $\mathrm{MND}=$ mild neurologic disorder; MND = mild neurocognitive disorder

B y $2013,>25 \%$ of the HIV-infected $\left(\mathrm{HIV}^{+}\right)$population in the United States was older than 55 years of age, a number projected to double by $2045 .{ }^{1}$ In this same population, the prevalence of HIV-associated neurocognitive disorders (HAND) in the United States may be up to $50 \%$, despite access to antiretroviral therapy. ${ }^{2}$ Cerebrovascular risk factors are prevalent in the aging

Received June 13, 2018; accepted after revision September 24.

From the Departments of Radiology and Biomedical Imaging (J.N., D.M., S.M.D., A.C., D.T.) and Neurology (J.H., V.V.), University of California at San Francisco, Zuckerberg San Francisco General Hospital and Trauma Center, San Francisco, California.

This study was supported by the American Foundation for AIDS Research (10930159-RSRL) and the University of California at San Francisco Resource Allocation Program (2014919).

Please address correspondence to Jared Narvid, MD, Department Radiology and Biomedical Imaging, University of California at San Francisco, Zuckerberg San Francisco General Hospital and Trauma Center, 1001 Potrero Ave, Room 1x57, SFGH Box 1325, San Francisco, CA 94110; e-mail: jared.narvid@ucsf.edu; @JaredNarvid

$\checkmark$ Indicates article with supplemental on-line photo.

http://dx.doi.org/10.3174/ajnr.A5902
$\mathrm{HIV}^{+}$population. These individuals face increased vascular comorbidities, including stroke, as well as cognitive deficits above those of the general population. ${ }^{3,4}$ While the pathobiologic basis of vascular and cognitive abnormalities in HAND is unclear, ${ }^{5}$ studies implicate injury to the neurovascular endothelium because HIV itself can induce endothelial dysfunction and capillary loss. ${ }^{6}$ Indeed, virally suppressed $\mathrm{HIV}^{+}$participants have gene-expression profiles suggesting neurovascular endothelial dysfunction. ${ }^{7-9}$ In this context, neuroimaging studies of $\mathrm{HIV}^{+}$ participants consistently demonstrated increased white matter intensities ${ }^{10-12}$ as well as altered cerebrovascular reactivity and autoregulation. ${ }^{13,14}$ Given that small-vessel ischemic disease is associated with reduced cerebral perfusion across time ${ }^{15}$ and hypoperfusion acts as precursor to volume loss in other neurodegenerative diseases, ${ }^{16,17}$ several investigators have found global cerebral perfusion abnormalities in the $\mathrm{HIV}^{+}$population. ${ }^{18-21}$ The current study evaluated the specific regional pattern of CBF abnormalities in older $\mathrm{HIV}^{+}$virally suppressed 


\begin{tabular}{|c|c|c|c|}
\hline & $\mathrm{HIV}^{+}$ & $\mathrm{HC}$ & $\mathrm{MCl}$ \\
\hline No. of participants & 19 & 13 & 19 \\
\hline HAND-MND diagnosis & 5 & - & - \\
\hline Age (mean) (minimum, maximum) (yr) & $66.31 \pm 3.09(61,72)$ & $69.28 \pm 4.73(60,74)$ & $65.83 \pm 4.19(59,74)$ \\
\hline Years of education (mean) & $16.74 \pm 2.26$ & $16.38 \pm 2.69$ & $16.47 \pm 2.93$ \\
\hline Sex & $18 \mathrm{M}, 1 \mathrm{~F}$ & $13 \mathrm{M}, 0 \mathrm{~F}$ & $19 \mathrm{M}, 0 \mathrm{~F}$ \\
\hline \multicolumn{4}{|l|}{ HIV variables } \\
\hline CD4 count (mean) (cells $/ \mathrm{mm}^{3}$ ) & $651.17 \pm 223.98$ & - & - \\
\hline CD4 nadir (mean) & $208.65 \pm 176.35$ & - & - \\
\hline Years since diagnosis (mean) & $21.5 \pm 7$ & - & - \\
\hline Undetectable viral load (\% of participants) & $100 \%$ & - & - \\
\hline \multicolumn{4}{|l|}{ Cerebrovascular risk factors } \\
\hline Myocardial infarct (No.) (\%) & $1(5.2 \%)$ & $0(0 \%)$ & $0(0.0 \%)$ \\
\hline Stroke (No.) (\%) & $0(0 \%)$ & $1(7.7 \%)$ & $0(0.0 \%)$ \\
\hline Diabetes (No.) (\%) & $2(10.5 \%)$ & $0(0.0 \%)$ & $0(0.0 \%)$ \\
\hline History of smoking (No.) (\%) & $10(52.6 \%)$ & $8(61.5 \%)$ & $8(42.1 \%)$ \\
\hline Hypertension history (No.) (\%) & $9(47.3 \%)$ & $4(30.8 \%)$ & $7(36.8 \%)$ \\
\hline Hypercholesterol history (No.) (\%) & $11(57.9 \%)$ & $11(84.6 \%)$ & $11(57.9 \%)$ \\
\hline
\end{tabular}

Note:- - indicates a variable which is inapplicable to the subject group.

participants using quantitative whole-brain arterial spin-labeling (ASL).

\section{MATERIALS AND METHODS \\ Participants}

This study was based on 19 available cross-sectional brain MRIs from older $\mathrm{HIV}^{+}$individuals enrolled in a larger cohort study of cognition in $\mathrm{HIV}^{+}$individuals older than 60 years of age (UCSF HIV Over 60 Cohort) that explored the relationship between HIV and aging. ${ }^{22}$ Five $\mathrm{HIV}^{+}$participants in the study (26.3\%) had mild neurocognitive disorder (MND) according to the Frascati criteria $^{23}$ a HAND subcategory. To compare ASL scans from $\mathrm{HIV}^{+}$participants and those with mild cognitive impairment (MCI), ${ }^{24}$ we matched participants with MCI and healthy controls (HC) to the HIV participants by age, sex, and education (Table). The matched brain scans of HC and MCI participants were obtained as a part of the Alzheimer Disease Neuroimaging Initiative cohort (ADNI-2), which included ASL (inclusion/exclusion criteria at www.adni-info.org). Participants with MCI had MiniMental State Examination scores of $>23$; objective memory loss as shown on scores on delayed recall of the Wechsler Memory Scale Logical Memory II (0.5-1.5 SDs below the normal mean); a Clinical Dementia Rating scale score of 0.5 in preserved activities of daily living; and absence of dementia. All HC had Mini-Mental State Examination scores of $>24$ and Clinical Dementia Rating scale scores of 0 . A $t$ test was used to compare the distributions of age and education between those who were HIV + and $\mathrm{HC}$ and between those who were $\mathrm{HIV}^{+}$and participants with MCI, after checking that populations were normally distributed (ShapiroWilk test) and had the same variance ( $F$ test).

Cerebrovascular risk factors such as diabetes, hypertension, and hypercholesterolemia were identified through either selfreport, medication list, or medical chart review and/or clinical data captured during the research visit. A 12-hour fasting serum level was obtained within 3 months of neuroimaging. Diabetes was defined as a fasting glucose level of $>125 \mathrm{mg} / \mathrm{dL}$ or a current clinical diagnosis from medical history. "Hypertension" was defined as having a systolic blood pressure of $>140$ $\mathrm{mm} \mathrm{Hg}$ or a diastolic blood pressure of $>90 \mathrm{~mm} \mathrm{Hg}$; and "hypercholesterolemia," as having a fasting low-density lipoprotein level of $>160 \mathrm{mg} / \mathrm{dL}$, a total cholesterol level of $>200$ $\mathrm{mg} / \mathrm{dL}$, and/or a current clinical diagnosis from medical history. "Smoking history" was defined as total tobacco use of $>100$ cigarettes in a lifetime.

\section{ASL Acquisition}

ASL was performed on a 3T MR imaging machine from a single vendor (Magnetom Skyra; Siemens, Erlangen, Germany) using a pulsed ASL method (quantitative imaging of perfusion using a single subtraction [QUIPPS] II with thin-slice T1 periodic saturation) with echo-planar imaging. ${ }^{25}$ Details of ASL data acquisition and processing are available on-line at adni.loni.usc.edu. Imaging parameters of the ASL scan used the ADNI-2 protocol: $\mathrm{FOV}=256 \mathrm{~mm}$, matrix $=64 \times 64, \mathrm{TR}=3400 \mathrm{~ms}, \mathrm{TE}=12 \mathrm{~ms}$, $\mathrm{TI}=700 \mathrm{~ms}$, total transit time of the spins $(\mathrm{T} 2)=1900 \mathrm{~ms}$, tag thickness $=100 \mathrm{~mm}$, tag-to-proximal slice gap $=25.4 \mathrm{~mm}, 24$ axial slices, slice thickness $=4 \mathrm{~mm}$, time lag between slices $=$ $2.5 \mathrm{~ms}$.

\section{ASL Preprocessing}

All ASL images were preprocessed using an extensively described pipeline. ${ }^{16,17}$ The ADNI investigator (D.T.) provided methods for quantitation of ASL data. Briefly, perfusionweighted images were computed by taking the difference between the mean-tagged and the mean-untagged ASL images. The first untagged ASL image (providing a full relaxed MR imaging signal) was used as a reference image of the water density and used to calibrate the ASL signal for computing CBF and to estimate the transformation to coregister ASL and structural MR imaging.

\section{Geometric Distortion-Correction and Structural-to-ASL Coregistration}

To accomplish registration between $\mathrm{CBF}$ and structural MR imaging maps, we augmented linear transformation with $9 \mathrm{df}$ based on normalized mutual information by a nonlinear registration approach based on total variance. ${ }^{26}$ 


\section{ASL Partial Volume Effect Correction}

The analysis aimed to measure blood flow in primarily gray matter tissue. To correct for gray/white matter partial volume effects, we adjusted the scaled perfusion-weighted image intensities according to a linear model of gray and white matter contributions to the ASL signal and on the basis of probabilistic segmentation of gray and white matter densities in each MR imaging voxel. Adjustments were made assuming a constant ratio between gray and white matter perfusion, and the scaled reference image was adjusted assuming constant ratios between gray matter and water, white matter and water, and CSF and water.

\section{Computation of CBF}

The scaled distortion-corrected coregistered and partial volumecorrected perfusion-weighted images were normalized to the reference image to express the ASL signal in physical units of arterial water density as $\mathrm{CBF}$ (Milliliters/100 $\mathrm{g} \times$ Minute).

\section{Structural MR Imaging Acquisition}

Structural MR imaging was also acquired within the ADNI-2 and ADNI Grand Opportunities protocols for registration purposes using a T1-weighted 3D MPRAGE sequence with the following acquisition parameters: $\mathrm{TR}=2300 \mathrm{~ms}, \mathrm{TE}=2.98 \mathrm{~ms}$, flip angle $=$ $9^{\circ}, \mathrm{FOV}=256 \mathrm{~mm}$, resolution $=1.1 \times 1.1 \times 1.2 \mathrm{~mm}^{3}$.

\section{ROIs}

FreeSurfer (surfer.nmr.mgh.harvard.edu) was used to generate anatomic ROI statistics for CBF and volume. Eighty-two brain regions from the left and right hemispheres were used in the analysis. Areas excluded from the analysis were the brain stem, corpus callosum, ventricles, CSF, cerebellum, choroid plexus, vessels, and optic chiasm, in both the left and right hemispheres. The left sensory cortex (postcentral gyrus) was considered a reference region where no changes were expected and was used to standardize the CBF values of the other brain regions. Moreover, CBF values were adjusted to account for volume differences among ROIs.

\section{Statistical Analysis}

Because the purpose of this study was to investigate a specific pattern in $\mathrm{CBF}$ impairment in $\mathrm{HIV}^{+}$participants compared with $\mathrm{HC}$ and those with MCI, the CBF by brain regions and possible confounder factors were used as predictors of participant diagnosis $\left(\mathrm{HIV}^{+}\right.$versus $\mathrm{HC}$ and $\mathrm{HIV}^{+}$versus $\left.\mathrm{MCI}\right)$ under the hypothesis that if $\mathrm{CBF}$ in specific brain regions can be used to predict the participant diagnosis, the CBF pattern differs between $\mathrm{HIV}^{+}$and $\mathrm{HC}$ (or MCI). All statistical analyses were performed in R (http:// www.r-project.org/). ${ }^{27} \mathrm{HC}$ and participants with MCI were frequency matched to $\mathrm{HIV}^{+}$participants as to age, sex, and education (2.5 SDs); however, age and education were still used in the analysis to adjust for any residual confounding.

The analyses were divided into 2 steps: 1 ) to create a predictive model to differentiate those $\mathrm{HIV}^{+}$from $\mathrm{HC}$ (respectively MCI) based on $\mathrm{CBF}$, and 2) to perform casual inference by identifying the key CBF brain regions that are different between the diagnostic groups. Because of these goals, the high dimensionality of the data ( 82 brain regions versus 32 participants), and the high degree of multicollinearity in the data, a generalized linear model via penalized maximum likelihood (GLMnet), ${ }^{28}$ was used to model the data. Given that our outcome variables were dichotomous $\left(\mathrm{HIV}^{+}\right.$versus $\mathrm{HC}$ and $\mathrm{HIV}^{+}$versus $\left.\mathrm{MCI}\right)$, the binomial family was used (logistic elastic net regression). This model uses a penalty value (L2 penalty term in the sum of squared error loss function) to shrink coefficients of correlated predictors toward each other. An additional parameter $(\alpha)$ combines the L1 penalty (number of zero coefficients) and L2 penalty terms. In the current modeling strategy, 10 -fold cross-validation was used to optimize these 2 hyperparameters. Additionally, all predictors were centered and scaled in the analysis. Missing data were compared between groups and brain regions to ensure that imputation was acceptable. $^{29}$

For both analyses $\left(\mathrm{HIV}^{+}\right.$versus $\mathrm{HC}$ and $\mathrm{HIV}^{+}$versus MCI), the data were resampled 500 times by bootstrapping to ensure stable parameter estimates from the GLMnet model. At each iteration of the bootstrap, the data were randomly split into a training and test set (60\% and $40 \%$, respectively) and a GLMnet model was fit using 10-fold cross-validation of the training set. The counts for each predictor, predictor coefficients, and accuracies of the model to predict participant classification on the left-out test set were collected at each iteration. Frequency bar charts of the accuracy, specificity, and sensitivity values were plotted.

Although elastic GLMnet regression does not generate a $P$ value, ${ }^{30}$ the mean coefficient value for each brain region and corresponding 95\% confidence intervals can be calculated from the bootstrap coefficient distribution. Thus, these were used to compute the odds ratios associated with each brain region by exponentiating the mean coefficients identified in the GLMnet model. The interpretation of an OR $>1$ is that an increase of CBF in the corresponding brain region resulted in an increase of the likelihood of being classified as an $\mathrm{HIV}^{+}$participant. The ORs were plotted as dot-whisker plots to inspect how each brain region contributed to the ability of the model to distinguish $\mathrm{HIV}^{+}$ participants from controls. Visual explanation of the processing pipeline used to obtain the model coefficients is see in On-line Fig 1.

\section{Effect of HAND Status on CBF}

As stated in the Table, 5 participants with HAND (categorized as MND) were among the $\mathrm{HIV}^{+}$participants. To study the effect of HAND diagnosis on the results of the GLMnet regression for all $\mathrm{HIV}^{+}$participants' CBF (sensitivity analysis), a GLMnet model was computed and bootstrapped 50 times with the whole cohort and again excluding the 5 participants with HAND-MND. To study the differences between the 2 sets of models, the mean coefficients for each brain region and demographic predictor were computed for each technique (including or excluding participants with HAND-MND $\mathrm{HIV}^{+}$) and the differences in mean coefficients were compared with the SD associated with the corresponding predictor.

\section{RESULTS}

\section{Participant Characteristics}

Nineteen $\mathrm{HIV}^{+}, 13$ age- and education frequency-matched $\mathrm{HC}$ and 19 age- and education frequency-matched participants with MCI were included in these analyses (Table). For all participants, 


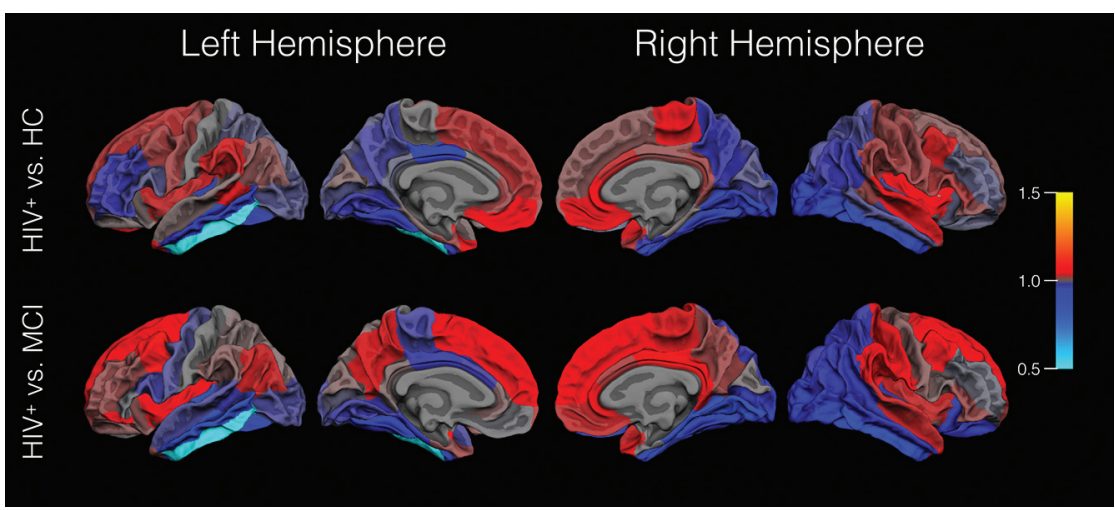

FIG 1. Odds ratio from the GLMnet model averaged over 500 bootstrap iterations. Reductions in $\mathrm{CBF}$ in bilateral temporal, occipital, and posterior cingulate regions best distinguish $\mathrm{HIV}^{+}$participants from $\mathrm{HC}$ (blue, hypoperfusion). This same pattern of reduced CBF distinguishes $\mathrm{HIV}^{+}$from $\mathrm{MCl}$, while the latter shows comparative reductions in frontal CBF (red, hyperperfusion in $\mathrm{HIV}^{+}$ participants relative to $\mathrm{MCl}$ ).

age ranged from 59 to 74 years old, and education ranged from 12 to 21 years. All $\mathrm{HIV}^{+}$participants had suppressed plasma HIV RNA levels. There were $98 \%$ male participants in the whole cohort (Table). The $\mathrm{HIV}^{+}$group included 5 participants with HANDMND meeting the criteria for MND, and 14 cognitively healthy $\mathrm{HIV}^{+}$participants. No participants met the criteria for HIV-associated dementia or asymptomatic neurocognitive impairment. Cardiovascular and cerebrovascular risk factors were present within the $\mathrm{HIV}^{+}$group in proportions similar to those in the $\mathrm{HC}$ and MCI groups (Table).

\section{ROI CBF Alterations}

Odds ratios from the GLMnet analysis were evaluated for 82 brains regions. Figure 1 shows the whole-brain patterns of $\mathrm{CBF}$ changes in each diagnostic group $\left(\mathrm{HIV}^{+}, \mathrm{HC}, \mathrm{MCI}\right)$. Cold colors reflect hypoperfusion used to accurately distinguish $\mathrm{HIV}^{+}$participants from the second group (HC or participants with MCI). Hot colors reflect relative hyperperfusion in $\mathrm{HIV}^{+}$participants compared with the second group. $\mathrm{HIV}^{+}$participants are distinguished by bilateral occipital, posterior cingulate, and bilateral temporal lobe hypoperfusion relative to HC. Compared with those with $\mathrm{MCI}, \mathrm{HIV}^{+}$participants are distinguished by a pattern of bilateral temporal, posterior cingulate, and occipital hypoperfusion and bilateral frontoinsular hyperperfusion. The complete dataset of regional odds ratios is shown in Fig 2.

\section{Performances of the Elastic Net Regression}

The elastic net regression performed prediction of $\mathrm{HIV}^{+}$versus HC with a median accuracy of 0.75 , a median sensitivity of 0.87 , and a median specificity of 0.57 ; and a prediction of $\mathrm{HIV}^{+}$versus MCI with a median accuracy of 0.73 , a median sensitivity of 0.75 , and a median specificity of 0.71 . The frequency distributions of accuracy, sensitivity, and specificity over the bootstrap are shown in Fig 3.

\section{Effect of HAND Status on CBF}

On-line Fig 2 shows the difference of mean coefficients in the GLMnet models for regional CBF when including or excluding HAND-MND from $\mathrm{HIV}^{+}$participants (gray bars) compared with the SD associated with the corresponding predictor (black lines) for the 2 models ( $\mathrm{HIV}^{+}$versus $\mathrm{HC}$ and $\mathrm{HIV}^{+}$versus MCI). A difference in mean coefficients smaller than the associated SD was observed for all brain regions and for both models, suggesting that the presence of participants with HAND-MND was not driving CBF abnormalities in specific regions or predictors for $\mathrm{HIV}^{+}$compared with $\mathrm{HC}$ or participants with MCI.

\section{DISCUSSION}

We demonstrate a significant effect of $\mathrm{HIV}^{+}$status on regional CBF compared with age-, sex-, and education-matched controls. Based on prior work, ${ }^{18-21}$ our primary hypothesis was that a pattern of regional cerebral hypoperfusion in HIV despite plasma viral suppression would discriminate $\mathrm{HIV}^{+}$participants from HIV-negative participants and from those with MCI. Our data suggest a pattern of hypoperfusion in HIV that involves bilateral temporal and occipital regions previously noted to be affected by longitudinal volumetric reductions ${ }^{22}$ in a larger sample of the same cohort (UCSF HIV Over 60 Cohort). Moreover, regional cortical thickness reductions in temporal and occipital regions were related to poor neuropsychological performance in other cohorts of treated virally suppressed $\mathrm{HIV}^{+}$participants. ${ }^{31}$ Most important, given volume correction as part of our data, our findings cannot be explained by structural variations in these participants; the reduction in CBF reflects perfusion within volumes rather than the effects of volume loss. These data and distribution of abnormalities are in keeping with a prior ASL analysis that was restricted to a limited set of brain slices. ${ }^{18}$ These results suggested that an older cohort of $\mathrm{HIV}^{+}$participants demonstrated a CBF profile that might suggest the diagnosis of HAND rather than MCI.

Few studies have evaluated quantitative CBF changes in viralsuppressed $\mathrm{HIV}^{+}$participants. Ances et al ${ }^{18}$ evaluated mostly untreated $\mathrm{HIV}^{+}$participants with ASL without whole-brain coverage due to technical limitations of the available ASL technology at the time. Nonetheless, they found reduced CBF in bilateral occipital volumes and the lenticular nucleus. This prior work represents a profile of a different population, primarily of a young age and detectable viral load. More recently, Su et $\mathrm{al}^{23}$ evaluated mean gray matter $\mathrm{CBF}$ using whole-brain ROIs, demonstrating reduced $\mathrm{CBF}_{\text {in }} \mathrm{HIV}^{+}$participants on antiretroviral therapy. Most important, the current study includes assessment of the vascular comorbidities that often accompany $\mathrm{HIV}^{+}$status. ${ }^{32}$ In our study, $\mathrm{HIV}^{+}$ participants demonstrated similar rates of smoking, hypertension, and hypercholesterolemia compared with matched healthy controls and participants with $\mathrm{MCI}$, though $2 \mathrm{HIV}^{+}$participants had a history of diabetes.

As the $\mathrm{HIV}^{+}$population reaches geriatric age, differentiating HAND from early-stage Alzheimer disease has become a pressing HIV concern. ${ }^{24} \mathrm{HIV}$-infected elders have higher rates of meeting the criteria for MCI, an intermediate state between typical cognitive aging and dementia in HIV-uninfected patient populations. ${ }^{33}$ Comprehensive cognitive testing may discriminate the cognitive 


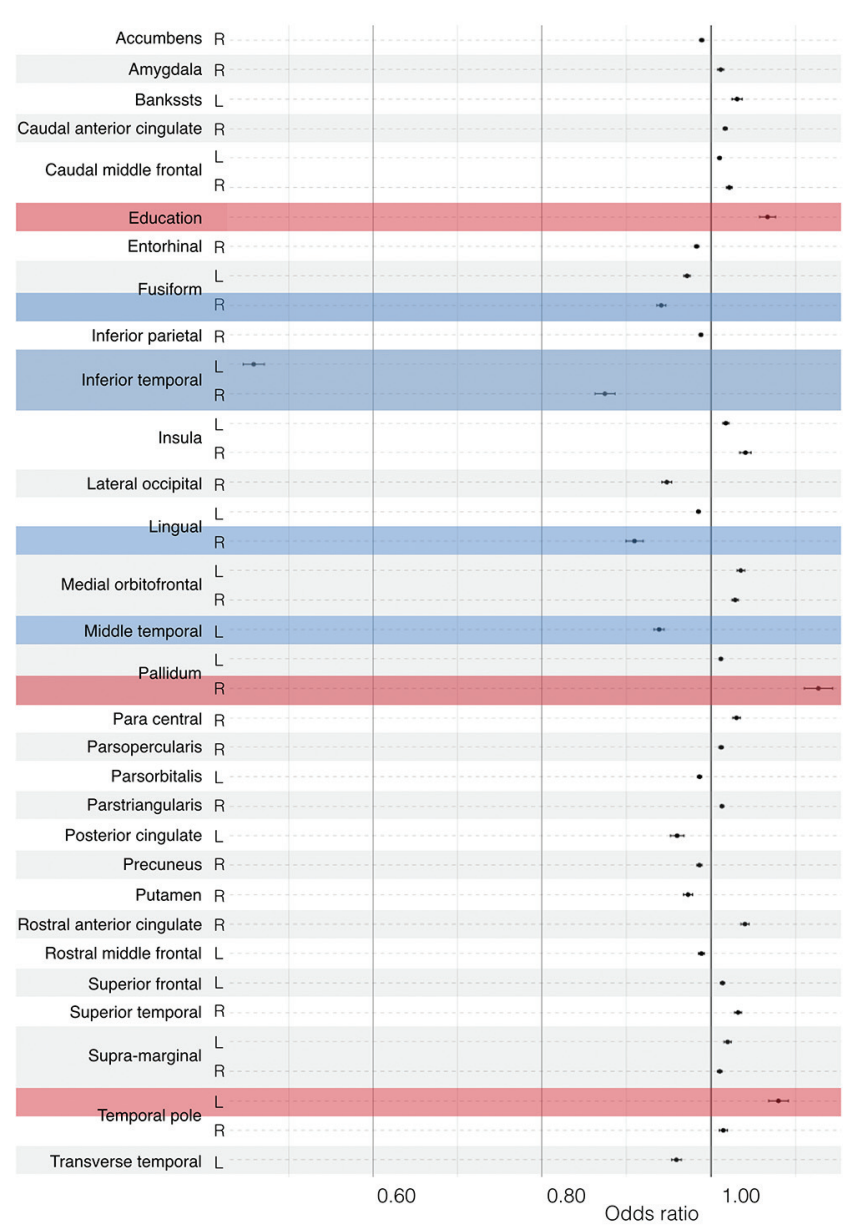

HIV+ vs. HC

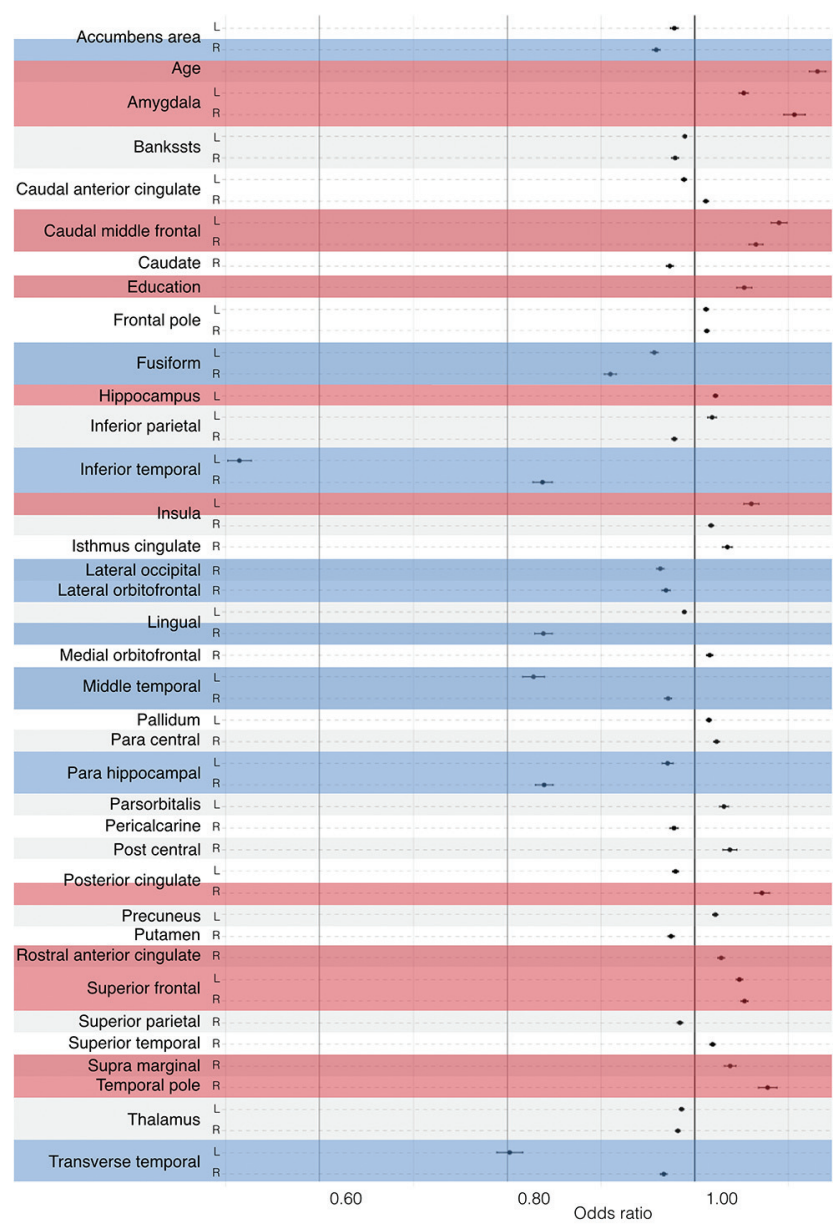

$\mathrm{HIV}+$ vs. $\mathrm{MCl}$

FIG 2. Odds ratios for each brain region and confounding factors in the GLMnet model, obtained by exponentiating the model coefficients averaged over a bootstrap (500 iterations). The regions used in the model in $>25 \%$ bootstrap iterations are highlighted in blue for the region hypoperfused in $\mathrm{HIV}^{+}$participants and in red for regions hyperperfused in $\mathrm{HIV}^{+}$participants compared with $\mathrm{HC}$ or those with $\mathrm{MCl}$. $\mathrm{HIV}^{+}$ participants demonstrate relative hypoperfusion in bilateral temporal and occipital regions and relative frontoinsular hyperperfusion.

phenotypes of mild HAND from those of MCI due to Alzheimer disease with reasonable accuracy. ${ }^{24}$ Here we evaluated whether CBF measurements may add specificity to the evaluation of cognitive symptoms in elderly $\mathrm{HIV}^{+}$participants. Our data suggest that $\mathrm{CBF}$ differences more accurately distinguish $\mathrm{HIV}^{+}$participants from $\mathrm{HC}$ compared with $\mathrm{HIV}^{+}$participants from those with MCI. Given temporal and parietal CBF changes within the Alzheimer disease spectrum, ${ }^{17}$ these findings are not surprising and explain the reduced sensitivity. However, when present, the abnormal CBF pattern in $\mathrm{HIV}^{+}$participants is quite specific for the diagnosis of $\mathrm{HIV}^{+}$compared with MCI. These findings suggest that ASL CBF measurements demonstrate appropriate test characteristics for a confirmatory study after neuropsychological evaluation in elderly $\mathrm{HIV}^{+}$participants. Future work to evaluate the discriminative power of a combined cognitive and ASL CBF dataset is needed.

Sensitivity analyses were completed to examine whether CBF changes in patients with HAND-MND were driving CBF differences when comparing all $\mathrm{HIV}^{+}$participants with other groups. We evaluated whether the HAND-MND subgroup showed regional CBF differences greater than the SD of all others by comparing the mean coefficients in the GLMnet analyses. Given that the differences between the mean coefficients of each region between HAND-MND and others were much smaller compared with the SD, the presence of HAND-MND is unlikely to explain $\mathrm{CBF}$ abnormalities among all $\mathrm{HIV}^{+}$participants.

The mechanisms for the observed CBF alterations within $\mathrm{HIV}^{+}$participants remain an area of active investigation. While many mechanisms might explain the interactive effects of age and HIV on the CNS, data suggest that immune activation may induce and promote cerebrovascular inflammation. ${ }^{34}$

Strengths of the current study include the comparison of our cohort of $\mathrm{HIV}^{+}$participants with a comparable HIV-uninfected control group and MCI group, under uniforming imaging and analysis conditions i.e. using the same ASL sequence, scanner and scanning parameters and analysis pipeline. In the existing literature, CBF measurement techniques often vary considerably among different study cohorts. ${ }^{18,20,21}$ In addition, our analysis required no a priori hypothesis with regard to a specific region of abnormality. Another strength is the relative similarity of cerebrovascular risk factors between our $\mathrm{HIV}^{+}$participants and comparison groups, a frequent confounder of studies of CBF and vascular disease in HIV. However, these results should be interpreted cautiously along with the limitations of the study. First, because of 

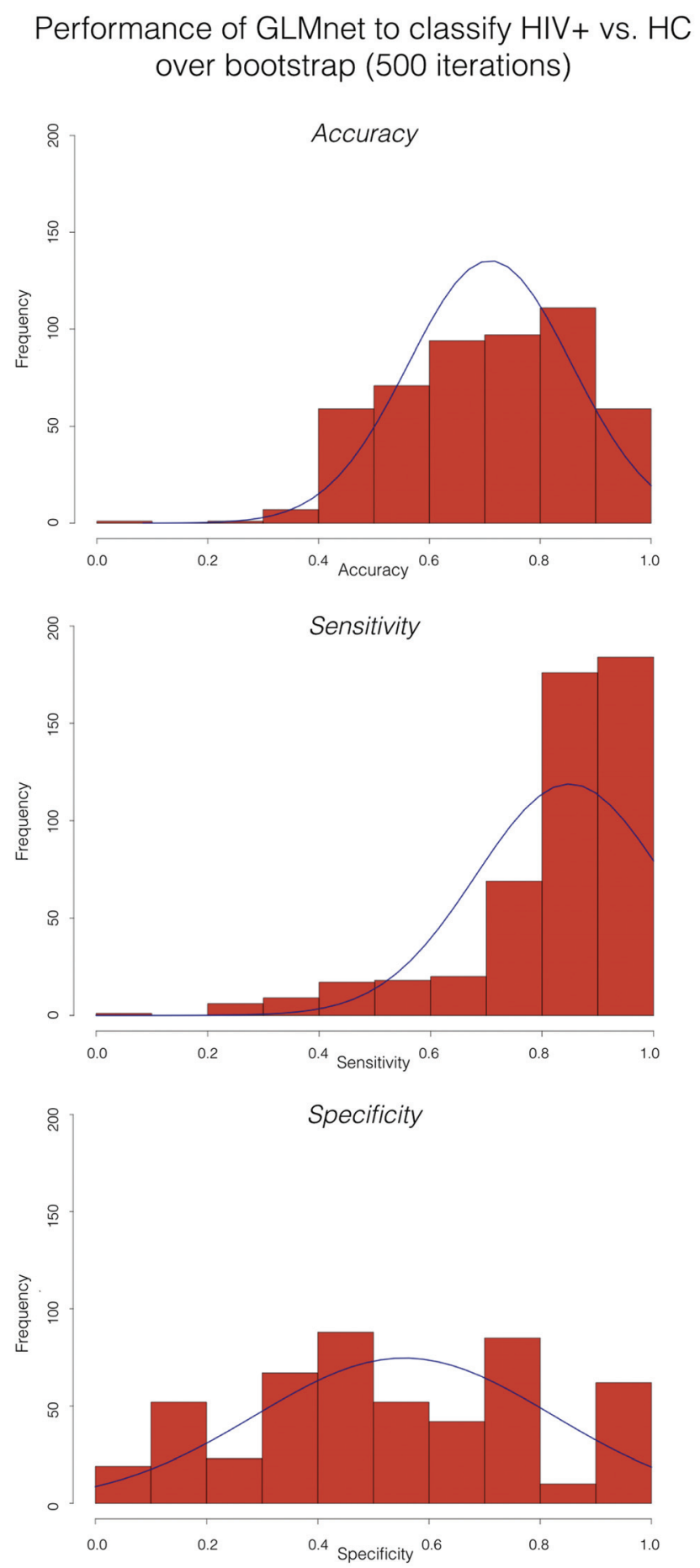

Performance of GLMnet to classify HIV+ vs. MCI over bootstrap (500 iterations)
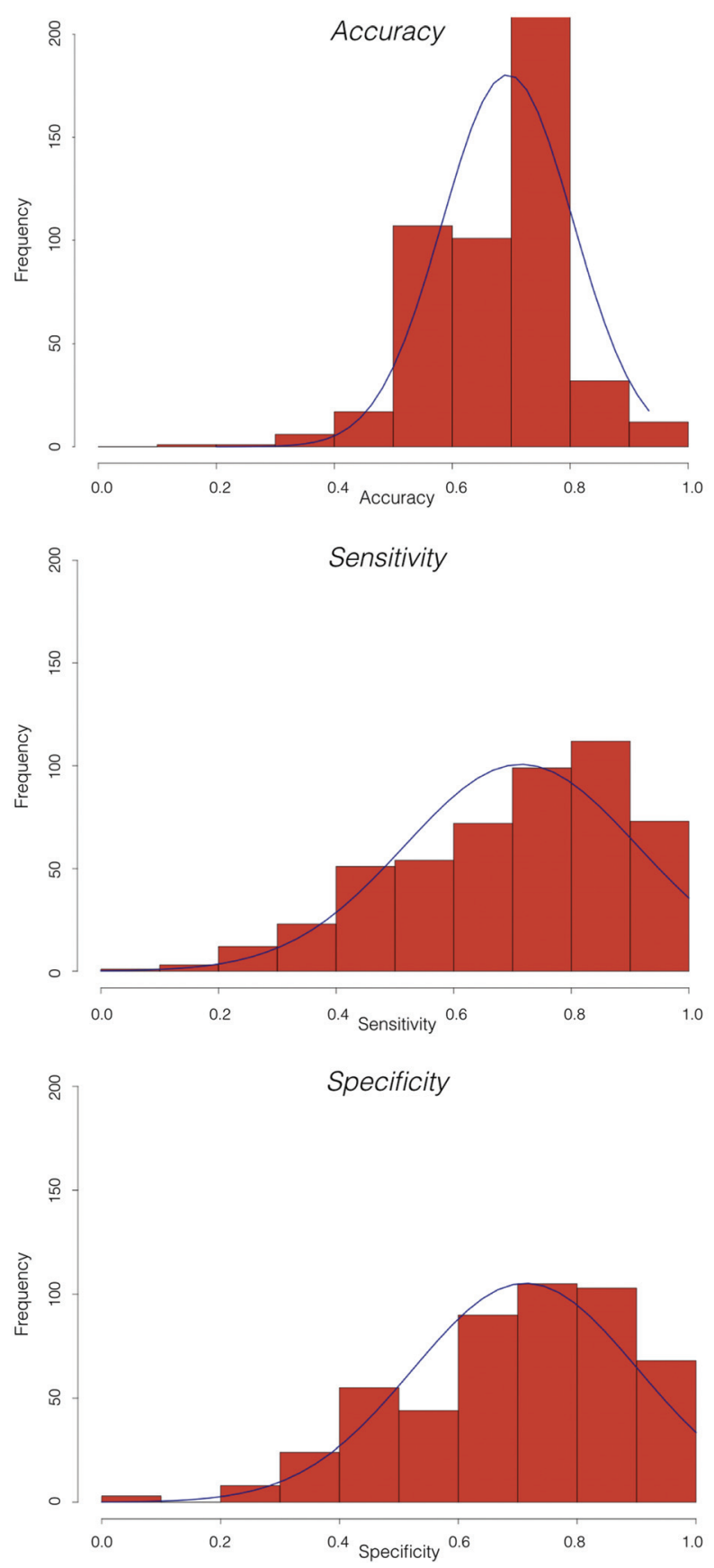

FIG 3. Histogram of the frequency distribution of accuracy, sensitivity, and specificity over the bootstrap (500 iterations) for the proposed GLMnet models.

the modest sample size, particularly in terms of uninfected controls, our findings are preliminary and need to be verified in a larger independent study. Second, this study presents an almost entirely male population, which is particularly challenging given data suggesting cerebrovascular risk in HIV may be higher in woman. ${ }^{35}$ Finally, the age of $\mathrm{HIV}^{+}$participants reflects frequent seroconversion before the generalization of combination antiretroviral therapy, and this finding may not be generalizable to younger post-antiretroviral therapy populations.

\section{CONCLUSIONS}

When we accounted for age, education, sex, and vascular risk factors, $\mathrm{HIV}^{+}$participants demonstrated alterations in regional cerebral perfusion, including hypoperfusion of bilateral temporal, parietal, and occipital volumes compared with both clinically healthy individuals and those with MCI. Our data also add to previous reports that MR imaging-measured CBF has diagnostic ability in comparison with cognitive assessment in distinguishing HAND-MND from MCI. 
Disclosures: Jared Narvid-RELATED: Grant: American Foundation for AIDS Research, Comments: This research was supported by a grant from the amfAR. * Sara M. Dupont-RELATED: Grant: AmFAR.* Andrew Callen-UNRELATED: Grants/Grants Pending: University of California at San Francisco Department of Radiology and Biomedical Imaging, Comments: Departmental Seed Grant of $\$ 10,000$ for the investigation of cerebral vasoreactivity in HIV-infected virally suppressed individuals using a novel MR perfusion technique. Victor Valcour-UNRELATED: Consultancy: ViiV Healthcare, Comments: consultation around HIV and aging; Employment: University of California at San Francisco, Comments: academic researcher; Grants/ Grants Pending: National Institutes of Health, Comments: research support*; Payment for Lectures Including Service on Speakers Bureaus: IAS-USA, Comments: Continuing Medical Education training on HIV. *Money paid to the institution.

\section{REFERENCES}

1. Hood JE, Golden MR, Hughes JP, et al. Projected demographic composition of the United States population of people living with diagnosed HIV. AIDS Care 2017;29:1543-50 CrossRef Medline

2. Heaton RK, Clifford DB, Franklin DR Jr, et al; CHARTER Group. HIV-associated neurocognitive disorders persist in the era of potent antiretroviral therapy: CHARTER study. Neurology 2010;75: 2087-96 CrossRef Medline

3. Hasse B, Ledergerber B, Furrer H, et al; Swiss HIV Cohort Study. Morbidity and aging in HIV-infected persons: the Swiss HIV cohort study. Clin Infect Dis 2011;53:1130-39 CrossRef Medline

4. Chow FC. HIV infection, vascular disease, and stroke. Semin Neurol 2014;34:35-46 CrossRef Medline

5. Hsue PY, Deeks SG, Hunt PW. Immunologic basis of cardiovascular disease in HIV-infected adults. J Infect Dis 2012;205(Suppl 3): S375-82 CrossRef Medline

6. Silva JN, Polesskaya O, Wei HS, et al. Chronic central nervous system expression of HIV-1 Tat leads to accelerated rarefaction of neocortical capillaries and loss of red blood cell velocity heterogeneity. Microcirculation 2014;21:664-76 CrossRef Medline

7. Jia P, Zhao Z, Hulgan T, et al; CHARTER Study Group. Genomewide association study of $\mathrm{HIV}$-associated neurocognitive disorder (HAND): a CHARTER group study. Am J Med Genet B Neuropsychiatr Genet 2017;174:413-26 CrossRef Medline

8. Gelman BB, Chen T, Lisinicchia JG, et al; National NeuroAIDS Tissue Consortium. The National NeuroAIDS Tissue Consortium brain gene array: two types of HIV-associated neurocognitive impairment. PLoS One 2012;7:e46178 CrossRef Medline

9. Gelman BB. Neuropathology of HAND with suppressive antiretroviral therapy: encephalitis and neurodegeneration reconsidered. Curr HIV/AIDS Rep 2015;12:272-79 CrossRef Medline

10. Nakamoto BK, Jahanshad N, McMurtray A, et al. Cerebrovascular risk factors and brain microstructural abnormalities on diffusion tensor images in HIV-infected individuals. J Neurovirol 2012;18: 303-12 CrossRef Medline

11. Watson C, Busovaca E, Foley JM, et al. White matter hyperintensities correlate to cognition and fiber tract integrity in older adults with HIV. J Neurovirol 2017;23:422-29 CrossRef Medline

12. Su T, Wit FW, Caan MW, et al; AGEhIV Cohort Study. White matter hyperintensities in relation to cognition in HIV-infected men with sustained suppressed viral load on combination antiretroviral therapy. AIDS 2016;30:2329-39 CrossRef Medline

13. Chow FC, Boscardin WJ, Mills C, et al. Cerebral vasoreactivity is impaired in treated, virally suppressed HIV-infected individuals. AIDS 2016;30:45-55 CrossRef Medline

14. Chow FC, Li Y, Hu Y, et al. Relationship between HIV infection, antiretroviral therapy, inflammatory markers, and cerebrovascular endothelial function among adults in urban China. J Acquir Immune Defic Syndr 2017;74:339-46 CrossRef Medline

15. van der Veen PH, Muller M, Vincken KL, et al; SMART Study Group. Longitudinal relationship between cerebral small-vessel disease and cerebral blood flow: the second manifestations of arterial disease-magnetic resonance study. Stroke 2015;46:1233-38 CrossRef Medline

16. Mattsson N, Tosun D, Insel PS, et al; Alzheimer's Disease Neuroimaging Initiative. Association of brain amyloid- $\boldsymbol{\beta}$ with cerebral per- fusion and structure in Alzheimer's disease and mild cognitive impairment. Brain 2014;137:1550-61 CrossRef Medline

17. Tosun D, Schuff N, Jagust W, et al; Alzheimer's Disease Neuroimag ing Initiative. Discriminative power of arterial spin labeling magnetic resonance imaging and $18 \mathrm{~F}$-fluorodeoxyglucose positron emission tomography changes for amyloid- $\beta$-positive subjects in the Alzheimer's disease continuum. Neurodegener Dis 2016;16: 87-94 CrossRef Medline

18. Ances BM, Sisti D, Vaida F, et al; HNRC group. Resting cerebral blood flow: a potential biomarker of the effects of HIV in the brain. Neurology 2009;73:702-08 CrossRef Medline

19. Su T, Mutsaerts HJ, Caan MW, et al; AGEhIV Cohort Study. Cerebral blood flow and cognitive function in HIV-infected men with sustained suppressed viremia on combination antiretroviral therapy. AIDS 2017;31:847-56 CrossRef Medline

20. Towgood KJ, Pitkanen M, Kulasegaram R, et al. Regional cerebral blood flow and FDG uptake in asymptomatic HIV-1 men. Hum Brain Mapp 2013;34:2484-93 CrossRef Medline

21. Sen S, An H, Menezes P, et al. Increased cortical cerebral blood flow in asymptomatic human immunodeficiency virus-infected subjects. J Stroke Cerebrovasc Dis 2016;25:1891-95 CrossRef Medline

22. Clifford KM, Samboju V, Cobigo Y, et al. Progressive brain atrophy despite persistent viral suppression in HIV patients older than $\mathbf{6 0}$ years. J Acquir Immune Defic Syndr 2017;76:289-97 CrossRef Medline

23. Antinori A, Arendt G, Becker JT, et al. Updated research nosology for HIV-associated neurocognitive disorders. Neurology 2007;69: 1789-99 CrossRef Medline

24. Milanini B, Valcour V. Differentiating HIV-associated neurocognitive disorders from Alzheimer's disease: an emerging issue in geriatric NeuroHIV. Curr HIV/AIDS Rep 2017;14:123-32 CrossRef Medline

25. Luh WM, Wong EC, Bandettini PA, et al. QUIPSS II with thin-slice TI1 periodic saturation: a method for improving accuracy of quantitative perfusion imaging using pulsed arterial spin labeling. Magn Reson Med 1999;41:1246-54 Medline

26. Tao R, Fletcher PT, Gerber S, et al. A variational image-based approach to the correction of susceptibility artifacts in the alignment of diffusion weighted and structural MRI. Inf Process Med Imaging 2009;21:664-75 Medline

27. R Development Core Team. $\mathrm{R}$ foundation for statistical computing. https://www.r-project.org/.

28. Friedman J, Hastie T, Tibshirani R. Regularization paths for generalized linear models via coordinate descent. J Stat Softw 2010;33; 1-22 Medline

29. van Buuren S, Groothuis-Oudshoorn K. MICE: multivariate imputation by chained equations in R. J Stat Softw 2011;45. https://www.jstatsoft.org/article/view/v045i03.

30. Frigessi A, Bühlmann P, Glad IK, et al. Statistical Analysis for HighDimensional Data: The Abel Symposium 2014. New York: Springer; 2016

31. Sanford R, Fernandez Cruz AL, Scott SC, et al. Regionally specific brain volumetric and cortical thickness changes in HIV-infected patients in the HAART era. J Acquir Immune Defic Syndr 2017;74: 563-70 CrossRef Medline

32. Kendall CE, Wong J, Taljaard M, et al. A cross-sectional, populationbased study measuring comorbidity among people living with HIV in Ontario. BMC Public Health 2014;14:161 CrossRef Medline

33. Sheppard DP, Iudicello JE, Bondi MW, et al. Elevated rates of mild cognitive impairment in HIV disease. J Neurovirol 2015;21:576-84 CrossRef Medline

34. Hong S, Banks WA. Role of the immune system in HIV-associated neuroinflammation and neurocognitive implications. Brain Behav Immun 2015;45;1-12 CrossRef Medline

35. Chow FC, Regan S, Zanni MV, et al. Elevated ischemic stroke risk among women living with HIV infection. AIDS 2018;32:59-67 CrossRef Medline 